\title{
The third arm: the surgeon's friend in the management of intraoperative complications in robotic-assisted rectal surgery
}

\author{
M. Milone ${ }^{1,2} \cdot$ M. Manigrasso $^{1} \cdot$ M. Gandini $^{1}$-S. Aprea ${ }^{1} \cdot$ G. D. De Palma ${ }^{1}$
}

Received: 23 July 2019 / Accepted: 14 August 2019 / Published online: 27 August 2019

(c) Springer Nature Switzerland AG 2019

Different studies have shown that robotic-assisted rectal surgery compared to traditional laparoscopic surgery is associated with less blood loss and a better preservation of the pelvic autonomic nerves that is fundamental in rectal surgery to avoid anterior resection syndrome [1,2].

Advantages of robotic technology should facilitate rectal cancer resection and some authors have demonstrated that conversion rates from robotic to open surgery, especially in low anterior resection, are lower than from laparoscopic to open surgery [3].

Therefore, robotic-assisted surgery is gaining popularity in the treatment of rectal cancer.

The attached online video shows a case of rectal cancer treated with a robotic approach, in which acute bleeding from the inferior mesenteric artery (IMA) occurred.

With the help of the third robotic arm, the bleeding was temporarily stopped, making it possible for the procedure to continue without any necessity of conversion. Moreover, the surgeon could go on using the other instruments without any discomfort.

After the dissection of the vascular plane along the IMA, and correct lymphadenectomy, the IMA could be clipped at its origin, definitively stopping the bleeding.

The intervention was completed without conversion and the patient was discharged on the 4th postoperative day without any complications.

Electronic supplementary material The online version of this article (https://doi.org/10.1007/s10151-019-02067-x) contains supplementary material, which is available to authorized users.

M. Milone

milone.marco.md@gmail.com

1 Department of Clinical Medicine and Surgery, Federico II

University of Naples, Naples, Italy

2 Department of Gastroenterology Endocrinology and Surgical Endoscopy, Federico II University of Naples, Via Sergio

Pansini, 5, 80131 Naples, Italy
Author contributions MM: conception and design of the article; MM, MG, and SA: acquisition of the data; GDDeP: critical revisions and final approval.

\section{Compliance with ethical standards}

Conflict of interest The authors declare that they have no conflict of interest.

Ethical approval Ethical approval is not required.

Human and animal rights This article does not contain any studies with human participants or animals performed by any of the authors.

Informed consent Written informed consent for publication of his clinical images was obtained by the patient.

\section{References}

1. Liu WH, Yan PJ, Hu DP, Jin PH, Lv YC, Liu R, Yang XF, Yang KH, Guo TK (2019) Short-term outcomes of robotic versus laparoscopic total mesorectal excision for rectal cancer: a cohort study. Am Surg 85(3):294-302

2. Jayne D, Pigazzi A, Marshall H, Croft J, Corrigan N, Copeland J, Quirke P, West N, Rautio T, Thomassen N, Tilney H, Gudgeon M, Bianchi PP, Edlin R, Hulme C, Brown J (2017) Effect of robotic-assisted vs conventional laparoscopic surgery on risk of conversion to open laparotomy among patients undergoing resection for rectal cancer: the ROLARR randomized clinical trial. JAMA 318(16):1569-1580

3. Wen-Xi Wu, Sun Yao-Min, Hua Yi-Bin, Shen Li-Zong (2004) Laparoscopic versus conventional open resection of rectal carcinoma: a clinical comparative study. World J Gastroenterol 10(8):1167-1170

Publisher's Note Springer Nature remains neutral with regard to jurisdictional claims in published maps and institutional affiliations. 